\title{
PERIODISMO ILUSTRADO EN MURCIA: EL CORREO LITERARIO (1792-1795)
}

\author{
Por Cayetano MAS GALVAÑ
}

Un periódico aparecido en Murcia durante la última década del siglo XVIII constituye el objeto de esta comunicación (1). Se trata del Correo Literario de Murcia, publicado entre 1792 y 1795 , al que consideramos típico exponente del periodismo ilustrado provinciano. Por sí sola, esta publicación periódica merecería una monografía de cierta extensión, dado su propio volumen. Además, los archivos murcianos no la conservan en su integridad (2). De ahí que hayamos optado por efectuar una aproximación genérica, sin perjuicio de ulteriores trabajos más detenidos y extensos, basándonos en los ejemplares publicados en el primer año de su existencia. Es decir, el período comprendido entre el 1 de septembre de 1792 y el 31 de agosto de 1793, en el que se publicaron los números 1 al 105.

De entrada, por tanto, tres términos se entrelazan: Ilustración, Murcia, periódico. En cuanto al carácter específico que presenta la Ilustración española, remitimos a los estudios generales que en las últimas décadas han venido a clarificarla bastante, subrayando sus rasgos diferenciales ${ }^{(3)}$. De hecho, los otros dos términos ejercen, en este caso, una presión restrictiva sobre la forma y el significado del primero. Como vamos a ver, el caso murciano tiene sus peculiaridades. Por otra parte, el periódico, en cuanto género literario, también presenta sus particularidades durante la época, que inciden sobre la forma del mensaje escrito. Nos colocamos, así pues, en una línea semejante a la sostenida en su comunicación a este mismo Congreso por el profesor Martínez Gomis. Junto a las grandes figuras de nuestra Ilustración (divulgadores o eruditos, Feijoo y Mayans, por citar las figuras prototípicas en ambos campos), es preciso, para reconstruir la realidad cultural de la época, recordar las modestas figuras a las que se refirió Sarrilh: «no vamos a evocar aquí a esas 'antorchas', sino ciertos hombres cultos, menos conocidos - nobles o burgueses, eclesiásticos o seglares-y aun a personas oscuras, cuyo recuerdo no ha sido conservado por la historia (...) hombres diseminados en muchas ciudades, pequeñas o grandes, y en algunos monasterios, que acogen favorablemente las nuevas ideas» ${ }^{(4)}$. 
El estudio de un periódico como éste, a pesar de sus limitaciones y también por ellas, constituye una buena vía por la que penetrar, más que en el sentido general de la Ilustración, en las actitudes de oposición que suscitó, en los mecanismos de penetración y asimilación de la ideología ilustrada entre las capas sociales receptoras de ese mensaje, en las modulaciones con las que éste fue aceptado en su divulgación, y en la pervivencia de elementos culturales anteriores. Hay en todo ello, desde cierto punto de vista, el problema de la «desvirtuación» del mensaje en su contenido y sentido prístino. Problema que en algunos aspectos enlaza con el de los «intermediarios culturales» y que halla un campo privilegiado de plasmación en la prensa, en sus redactores y en sus lectores.

Y es que el periódico - cualquier periódico-impone siempre sus particulares condicionantes. Como señala Guinard, el periódico es un reflejo e incluso un índice ${ }^{(5)}$, cuya existencia requiere una serie de condiciones: un grupo que considere necesaria la difusión de cierto tipo de informaciones y de ideas; un público potencial lo bastante coherente y numeroso como para que se esboce una opinión pública, de la que el grupo de informadores es -latu sensu - una emanación; $\mathrm{y}$, finalmente, resulta imprescindible la existencia de una «clase media» suficientemente numerosa y en posesión al menos de un embrión de su propia conciencia, y de unas condiciones generales (económicas y técnicas) que hagan viable la existencia del periódico ${ }^{(6)}$.

Sin olvidar la importancia que reviste el factor de vigilancia ejercido por las autoridades y la presión que impone la marcha de los acontecimientos políticos, interesa sobremanera subrayar que el periódico es un lugar de diálogo continuo entre el publicista y sus lectores: no se trata tanto de la correspondencia que los últimos pueden dirigir al periódico, como del consenso redactores-público del que el periódico es expresión necesaria. Desde este otro punto de vista, el periódico aporta informaciones preciosas acerca de los prejuicios, tabús, ideas y gustos del grupo al que se dirige mayoritariamente ${ }^{(7)}$.

Si la bibliografía existente sobre la prensa española del siglo XVIII dista aún bastante de cubrir suficientemente el tema, la carencia es casi total en cuanto se refiere al Correo Literario. En orden a las obras generales, fue omitido por Guinard, puesto que rebasaba los límites cronológicos de su estudio; R. Herr ha sido prácticamente el único que lo cita con cierta extensión y algunas imprecisiones ${ }^{(8)}$, por lo demás nimias; en cambio, María Dolores Saíz nunca lo menciona ${ }^{(9)}$. En consecuencia, la mayor cantidad de noticias relativas al Correo han de buscarse en las obras de prisma local ${ }^{(10)}$. Por descontado, no existe ninguna monografía consagrada al periódico que nos interesa.

El problema que plantea la aparición de un periódico en Murcia, que en principio podemos considerar ILUSTRADO, se explica sin grandes dificultades. Evidentemente, al margen de la necesaria existencia de las condiciones sociales, económicas y técnicas antes referidas, lo que cabe preguntarse es acerca de la propia existencia de la Ilustración en Murcia. Los grandes jalones de la llegada de estas corrientes a la capital murciana pueden ser fijados siguiendo el clásico esquema de Herr: Universidades, Sociedades Económicas y prensa como «conductos de la Ilustración» ${ }^{(11)}$. Murcia no contó con universidad propiamente dicha en esta 
época, pero el Seminario de San Fulgencio -de rancia tradición, fundado en 1592 por el obispo Sancho Dávila - vino a hacer las veces. Merced a la iniciativa del obispo Rubín de Celis y sus colaboradores, y al apoyo casi incondicional del gobierno, el Seminario fue sometido a una serie de reformas en sus planes de estudios desde 1774, y concesión de gracias en materia académica, que hicieron de él una Universidad de facto. En los años de mayor plenitud (precisamente la década de 1791-1800), llegó a contar con estudios de filosofía, teología, derecho civil y canónico. Un abundante alumnado, interno y externo, asistía a sus aulas (casi 500 alumnos por año), y en él podían recibirse los grados menores (Bachiller) en todas estas materias como si de cualquier otra Universidad se tratase ${ }^{(12)}$. Casi contemporáneamente, en 1778, era fundada la Sociedad Económica local ${ }^{(13)}$. Por último, 1792 registra la aparición de los primeros periódicos murcianos, el Diario y el Correo, inmediato sucesor del primero ${ }^{(14)}$. No deja de resultar sintomático el lapso transcurrido desde los años setenta hasta los noventa: lo entendemos como el tiempo necesario para la conformación de ese núcleo relativamente numeroso de individuos y de esa opinión pública necesaria como potencial público lector de un periódico como el de las características del que nos ocupa. Además, el trasvase de individuos entre las tres instituciones existe y es constatable, con lo cual, a través de ellas podríamos considerar establecido buena parte del círculo «ilustrado» murciano, tomando el término ilustrado en un sentido muy amplio y en buena medida desvirtuado. A este propósito, es necesario efectuar algunas precisiones: el Seminario, la Económica, y la prensa, más o menos adscritos a la Ilustración - no lo olvidemos, propugnada oficialmente - no son una misma cosa.

De las tres instituciones, el Seminario aglutina sin la menor sombra de duda, el núcleo más sólidamente formado en el pensamiento ilustrado, y por tanto, más combativo y próximo a los postulados teóricamente más «puros» de este conjunto de corrientes. Al propio tiempo, al Seminario y al conjunto de clérigos - seculares- que lo sustuvieron y dieron vida correspondió la misión de introducir en una sociedad murciana dominada culturalmente por elementos tradicionales -el clero regular, fundamentalmente- la «cuña» ilustrada. Erróneo sería intentar reducir la influencia del Seminario al clero. Las reformas de Rubín de Celis no fueron únicamente de orden académico-institucional. Unida a estas medidas se encontraba la reforma de los planes de estudios, abiertamente ilustrada. Y al Seminario acudieron, junto a quienes cursaban estudios aclesiásticos, la gran mayoría de los murcianos que, con suficientes recursos económicos, aspiraban a títulos civiles (filosofía y, sobre todo, derecho civil). De tal forma, podemos asegurar que una buena parte de los cuadros intelectuales y profesionales medios de Murcia y sus tierras habian pasado por el Seminario. No pretendemos reducir toda la cultura de la época en la capital del Segura a la obra del Colegio fulgentino. Subsistieron, y con enorme fuerza, las corrientes culturales anteriores, de cuño tradicional y barroco, mezcladas con intereses de prestigio y control social e ideológico. Tal es el origen del grupo de oposición al Seminario, integrado por los regulares, pero también por el propio Ayuntamiento en momentos cruciales, y que se mantuvo hasta bien entrado el siglo XIX. Tampoco podríamos decir que 
antes de la reforma del Seminario en 1774 no existiera difusión ilustrada en Murcia, ni que después ésta se centrase exclusivamente en el Colegio. Pero queremos insistir - puesto que hasta hoy no ha sido advertido- que sólo a partir de la institución fulgentina puede entenderse lo que fue la Ilustración, e incluso el primer liberalismo, en Murcia.

Es decir, que podemos distinguir, en la Murcia del momento, y con bastante claridad, los elementos más radicales (en el puro sentido etimológico del término) tanto dentro de la línea ilustrada como de la tradicional, sin que por ello caigamos en un reduccionismo simplista. En buena parte, es una disputa interclerical, entre el clero secular y el regular, de raíces bastante complejas y anteriores a la penetración, oficial, de las ideas ilustradas. Pero en el terreno estrictamente cultural, el error sería concebir un Seminario o un clero secular, aunque estuviese formado en él, absoluta o globalmente opuesto a unos regulares que, a su vez, serían todos un reducto de oposición antiilustrada. Por definición, el conocimiento cultural profundo es de carácter minoritario. En esa «desvirtuación» del mensaje, que en Murcia va de la mano del proceso de su difusión, que hemos dicho de carácter oficial, se encuentra la clave para comprender la existencia tanto del público como de la naturaleza misma del periódico que nos ocupa. Y también para entender lo que de injerto, de aceptación más bien formal que profunda, tuvo la Ilustración entre la mayoría de los miembros de las instituciones culturales y políticas murcianas. Pensamos especialmente en dos: el Ayuntamiento y la Sociedad Económica. De ambas es bien conocida la tibieza y la escasa comprensión manifestada corporativamente a los contenidos esenciales, al sentido profundo, de la Ilustración. Se abre así también la puerta para entender la debilidad intrínseca de los pilares sociológicos en que ésta se asentó en Murcia, y el cúmulo de posturas ambiguas, o de tonalidades intermedias, que advertimos caso por caso e individuo por individuo. El Correo será una clara expresión de ese contexto cultural y social de la capital del Segura a fines del siglo XVIII.

Como decíamos, el Correo... se publicó en Murcia entre el 1 de septiembre de 1792 y el 29 de diciembre de 1795. Salido de las prensas de la viuda de Felipe Teruel, el total de la publicación nos ha llegado encuadernado en 10 volúmenes en cuarto. Su periodicidad era bisemanal (sábado y martes) sostenida durante los treinta y nueve meses de publicación de modo ininterrumpido. Su carácter era misceláneo, como ya indicaba la propia portada, «sobre varios asuntos correspondientes a la política, física, moral, ciencias y artes», y en la misma, bajo la muy racional figura del triángulo equilátero, aparecía el significativo lema (tomado de la ep. 87 de Séneca) Otium sine litteris mors est. Los tomos, que responden al plan trazado por los editores, son cuatrimestrales. En cuanto a quiénes eran aquéllos, las iniciales $Z$. M. B. que figuran en los vértices del triángulo revelan su personalidad. Se trataba del erudito Miguel González Zamorano, del médico y poeta Francisco Mesegur y del sacerdote Luis Santiago Bado ${ }^{(15)}$. Conocemos varias publicaciones de los dos últimos, reseñadas en la Biblioteca del Murciano: unas obras satíricas y médicas de Meseguer ${ }^{(16)}$ y otras de Bado ${ }^{(17)}$, debidas a su condición de sacerdote y matemático. En especial, la figura de este último resulta interesante: aprendiz de escultor, sacerdote tras enviudar, profesor de matemáti- 
cas en las aulas de la Económica, prototípico clérigo ilustrado, con el advenimiento del liberalismo se convirtió en feroz detractor del nuevo sistema político y en apologeta del despotismo, pero manteniéndose en los criterios ilustrados. Por lo que se refiere a la intervención de los tres en la redacción de los artículos del periódico, resultan difíciles las atribuciones, dada la costumbre de no firmar, o de utilizar iniciales o variedad de seudónimos. Sin embargo, parece verosímil el juicio de Tejera ${ }^{(18)}$ según el cual los artículos de materia médica, literatura y costumbres pertenecerían a Meseguer; los de matemáticas, física, astronomía y moral, a Bado; y los de política, historia y afines, a Zamorano. Al mismo tiempo, los tres intervinieron como poetas y conjuntamente en la redacción de la historia de Murcia, cuya publicación emprendieron sin feliz término.

En relación con lo expuesto, hay que considerar, pues, las siguientes cuestiones:

1.-El carácter y la orientación del periódico.

2.-La situación cronológica y el contexto político.

3.-La situación local murciana.

Tres puntos totalmente imbricados. En cuanto al primero, y como hemos adelantado, falta una orientación hacia un campo específico. Sin sujetarse estrictamente a temas literarios, incorpora - lo que ya es en cierto modo novedoso-, artículos de tipo científico. Pero se dan escasas muestras de originalidad, usando no pocas veces de traducciones. Igualmente, el Correo Literario no se concibe desde una perspectiva crítica, al estilo de un Censor, que le coloque en dudosas posturas ante el sistema político y social. Por tanto, el principal interés es divulgativo, tanto desde la propia posición interdisciplinar de los editores, como desde la adaptación a aquella característica general que Guinard señala para la prensa española del siglo XVIII, al decir de la misma que se trataba de una prensa orientada. En España, los poderes no se limitaron, a través de los mecanismos de censura, a impedir las publicaciones que defendieran ideas políticas y religiosas juzgadas subversivas o amorales (caso francés), sino que además pretendieron que los periódicos fuesen esencialmente un instrumento útil, de educación y vulgarización, de progreso científico ${ }^{(19)}$. De ahí el carácter heterogéneo y misceláneo que atribuimos al Correo... No resulta extraño por cuanto la Ilustración, como ideología de una minoría social, contiene una visión del mundo y de su transformación de pretensiones globalizadoras. Aunque más adelante esbozaremos una clasificación temática, viene muy al caso recordar el Rasgo moral con el que, a modo de editorial y declaración de principios, se inaugura el primer número del Correo... Allí se dibujan, cierto que con brevedad, los valores más significativos de nuestra Ilustración: crítica de la ociosidad y de la impiedad, diferenciación entre una «mala filosofía», impía y materialista, y otra "verdadera», basada en la virtud, en el servicio útil a la sociedad y a la patria e inspirada en los valores cristianos. Escrito con brevedad, el Rasgo moral representa la punta del 'iceberg' ilustrado que va a ir emergiendo a lo largo de los números del Correo...

Además - y entramos con ello en el segundo punto- pesaban las limitaciones intrínsecas a todo periódico (el consenso redactores-público resulta ser un importante condicionante), la estricta legislación y la censura, que en este caso se 
ejercía sobre cada número, etc. Pero sobre todo cabe considerar el momento en el que aparece el Correo Literario. En esto, los tres redactores murcianos van a ser pioneros de la segunda generación de periódicos españoles del siglo XVIII. Aún antes de la caída de Floridablanca y, dato interesante, vigente la prohibición establecida sobre la circulación de periódicos no oficiales, el 1 de enero de 1792 ve la luz el primer periódico murciano, el Diario de Murcia ${ }^{(20)}$. Dadas las circunstancias, los contenidos del efímero periódico fueron bastante inocuos a la vez que trazados a semejanza de sus homólogos: comenzaba con la vida del santo del día, seguida por asuntos diversos, sátiras, fábulas, noticias y anuncios. Es el mismo esquema, aproximadamente, que después desarrollará el Correo... La aparición de otra publicación, el Censor del Diario de Murcia, junto con otras circunstancias poco claras al parecer en relación con la rivalidad existente entre los dos impresores locales ${ }^{(21)}$, pusieron fin a la vida del Diario en agosto de 1792. Para entonces, la prohibición de Moñino había sido levantada por Aranda. Inmediatamente después, el 1 de septiembre de ese año, los redactores del Diario emprenden la empresa del Correo, mucho más duradera. Ese mismo día, nació también el Diario de Sevilla. El propio Correo..., en su sección de notificas, decía admitir suscripciones a aquél y a los de Valencia y Barcelona. Como indica Herr ${ }^{(22)}$, esta segunda generación de periódicos, de comienzos muy circunspectos, mostró prontamente las mismas inclinaciones que la anterior. De hecho, la cantidad de páginas que el Correo... dedica durante 1792 al tema de la guerra contra Francia, a la apología nacional y a la exaltación del espíritu patriótico es considerable. Pero si esto es natural, lo mismo que las circunstancias políticas no permitían arriesgarse con artículos peligrosos, del conjunto de sus páginas comienza a destilarse cierto fondo crítico, el de la Ilustración que entraba en crisis, pasados los «idílicos» momentos del reinado del Carlos III (recordado con gran frecuencia). Tal situación, de manifestaciones muy complejas, resulta igualmente constatable en el Seminario de San Fulgencio. Las respuestas a la misma, entre los miembros del círculo ilustrado local, fueron muy variadas en esos y en años posteriores: liberales, afrancesados, absolutistas de diversa procedencia (como el propio Bado)... De momento, en el Correo... hay un esfuerzo claro por rechazar los extremos y mantenerse en la línea de la ilustración católica.

En cuanto al tercero de los puntos, el Correo... es producto de la favorable coyuntura general que Murcia vivió durante el siglo ${ }^{(23)}$ y de la introducción por otras instituciones de las ideas ilustradas, bien que sobre un tejido sociocultural bastante esclerotizacio y secularmente dominado por las órdenes religiosas (dominicos, franciscanos y jesuitas). También desde esta perspectiva se detecta la crisis. El esfuerzo de los redactores, quizá un tanto anacrónico, se orienta, tanto a difundir ese tipo concreto de Ilustración (en una fecha tan avanzada), como a proporcionar una herramienta para sostenerla. No en vano hemos de recordar que las fuerzas locales de oposición al Seminario, acalladas durante los tiempos de Carlos III, pronto lograrán ver barridas las reformas ilustradas que el mismo poder público había apoyado (en el período comprendido entre el «Cisma de Urquijo» y el «Plan Caballero», de 1807). El Correo... refleja algunos de los choques entre los dos grupos, con lo cual resulta mucho mayor la parte concedida 
en el periódico a la defensa de los principios ilustrados (la crítica, la utilización de la razón y de la experiencia, el combate contra la escolástica), que la correspondiente a la formulación de planteamientos que implicasen mayor profundidad (lo que sí ocurría en el Seminario). En definitiva, un periódico de tono bastante moderado, por no decir inocuo, con una débil retaguardia, y que, en este caso al menos, marca la frontera (con las consiguientes servidumbres), entre fuerzas antagónicas. Los elementos de la crisis se perfilan en la conciencia de los propios redactores, cuyo resultado hemos visto en la postura de Bado: de un lado, la lucha que acabamos de esbozar; de otro, la contemplación de los sucesos de Francia, que se juzgan producto de la Ilustración materialista de los philosophes.

En el cuadro I se ha intentando efectuar una evaluación cuantitativa que nos permita comprobar la implantación socioprofesional y geográfica del Correo literario. Nuestra investigación, que -como se ha dicho - se ha ocupado sólo del primer año de la existencia del periódico, revela que en dicho período (1-IX1791/31-VIII-1793; números 1 al 105) el Correo conoce una clara expansión. Se vendía a 4 rs. de vellón en Murcia, y a 7 fuera de la misma (franco portes), la suscripción mensual (números bisemanales de 8 páginas). En principio, podía adquirirse en Madrid, Sevilla, Valencia, Barcelona, Cartagena, Alicante y Orihuela. El procedimiento de las suscripciones, que parece haber sido utilizado con exclusividad, aseguraba la continuidad de la empresa sin exigir un excesivo desembolso de capital y con un mínimo de riesgo ${ }^{(24)}$. Parece, además, que los primeros suscriptores estaban asegurados incluso antes de la aparición del primer número ${ }^{(25)}$. Más tarde, el ámbito de venta se amplía, sin aumento de precio (lo que indica que los costes fijos estaban suficientemente cubiertos) a Cuenca, Valladolid, Burgos, Granada, Baeza, Jaén, Córdoba, Zaragoza, Lorca y Cádiz ${ }^{(26)}$. Lo mismo ocurre en cuanto al número de suscriptores: 253 en las primeras listas publicadas, al final del período a que nos referimos se contaba ya con 304 (27). Ambos datos se ampliarán posteriormente, e implican unas tiradas mínimas no inferiores a los 350-400 ejemplares por número (unos 700 por semana). Sin embargo, se trata de datos que deben manejarse con precaución, cuyo valor es simplemente aproximativo, en especial los referidos a suscriptores. Así, hemos detectado alguna que otra repetición en las personas relacionadas en las listas. Igualmente, desconocemos el número de cuántos pudieron darse de baja, aunque la insistencia de los editores sobre la necesidad de abono de las cuotas nos hace pensar a la vez en la existencia de no pocos casos y en el escaso margen de beneficio con que se trabajaba. En cuanto a los de fuera de Murcia y su región, en especial los de Madrid capital, algunos de ellos son murcianos residentes en la Corte. Las listas proporcionan siempre el nombre del suscriptor y la población en la que reside; desgraciadamente, en un tercio de los casos no se especifica la ocupación o el estado social. Como es lógico, en cuanto a la distribución geográfica de los suscriptores, el predominio corresponde a Murcia, con 150. Le sigue Andalucía (20 por 100,61 abonados) y, con sólo un 10 por 100 cada una, Castilla y Norte, Valencia y Cataluña. Por lo tanto, el Correo... fue un periódico bien difundido en la periferia mediterránea, máxime cuando consideramos que gran parte de los castellanos eran madrileños o residentes allí. 
La extracción sociológica tampoco revela sorpresas. En el cuadro se indica el total por grupos y su desglose regional. Salvo pequeñas alteraciones la jerarquización que se observa en Murcia no cambia en cuanto al total nacional se refiere. Dejando al margen el importante número de casos sin especificar (121), que seguramente $-\mathrm{y}$ a falta de posteriores cotejos - entendemos distribuible entre el resto de las categorías establecidas, ocupa el primer lugar el clero secular y las jerarquías eclesiásticas (clero catedralicio sobre todo), seguido de lejos por los funcionarios de la administración estatal, la nobleza y los comerciantes. El clero regular (especialmente mercedarios y agustinos), ocupa el último lugar destacable. Un esquema, pues, coincidente con los datos de que disponemos acerca de la sociología de la minoría ilustrada, difundida especialmente entre las capas medias y algunos sectores del clero y la nobleza, cuyas ausencias (médicos, juristas, etc.) con toda probabilidad habrá que adscribir a los casos no especificados.

El cuadro II pretende efectuar una clasificación por grupos de artículos, si bien algunos de los temas de fondo reaparecen constantemente en distintos grupos. En orden al método adoptado, se ha preferido efectuar un cómputo, también aproximativo, del número de páginas que abarca cada grupo, pese a los errores que ello comporta (63 páginas de error sobreestimativo de un total de 855 contabilizadas), causados por la presencia de las páginas que incluyen dos o más artículos (comienzo y fin de artículo). Consideramos preferible este sistema al de tener en cuenta únicamente el número de los destinados a tal o cual asunto, dado que mientras unos sólo ocupan una página o incluso menos, otros se prolongan a lo largo de sucesivos números. Siguiendo a Guinard ${ }^{(28)}$, así evitamos establecer una jerarquización temática a posteriori, basada en la relevancia que después han alcanzado a nuestros ojos, al tiempo que se reflejan más fielmente las preocupaciones y los gustos reales de los lectores del periódico.

Previo a toda clasificación, además, deben ser tenidos en cuenta aquellos presupuestos básicos antes señalados acerca del carácter ideológico de la Ilustración, de la posición interdisciplinar de los editores, y del carácter divulgativo, no monográfico, del periódico. No obstante, y atendiendo al número de páginas ocupadas, podemos ofrecer esta ordenación:

1.- Historia. Se publica una historia de Murcia, basada en las obras anteriores de Cascales y de Hermosino, pero desde planteamientos de crítica textual (174 páginas). Hubo de interrumpirse ante las quejas de un sector de los suscriptores, causadas por la lentitud y fárrgo de la publicación (escasamente 1-2 páginas por número). Aquí intentaron los editores beneficiarse de la ventaja que ofrecía el periódico al permitir la publicación de un libro sin elevados desembolsos iniciales de capital. Otras 33 páginas se dedican a diversos estudios basados en investigaciones históricas, normalmente con implicaciones de tipo moral (sobre las costumbres que observó la antigüedad en el nacimiento del hombre, reflexiones sobre la historia romana, y otras de esta índole). Cabe resaltas en este grupo dedicado a la historia dos hechos: el recurso a los autores del Siglo de Oro, y la utilización de una metodología crítica: se llega en ocasiones a censurar como poco críticos y excesivamente mitológicos a los historiadores contemporáneos. Este mismo hecho hizo demasiado farragosa la obra y también originó protestas del público ${ }^{(29)}$. 
2.-Composiciones en verso. Este grupo ocupa 184 páginas, en su mayoría fábulas y sátiras, letrillas y sonetos. Aunque, con fines clarificadores, podríamos asignar a las fábulas lo que Guinard denomina «lugares comunes morales» ${ }^{(30)} \mathrm{y}$ a las sátiras un contenido crítico más directo, lo cierto es que ambos tipos de composición comportan una clara censura de ciertos grupos sociales de la época y de sus costumbres: la ociosidad, los necios, las mujeres, los embusteros y usureros, el afeminamiento, los petimetres, la «vana erudición» de los «asnos letrados», la incompetencia en los cargos públicos y el abandono de las funciones sociales, los tiranos, el lujo, los médicos-charlatanes, y un largo etcétera son puestos en la picota. Sólo un reducido grupo de las composiciones de este grupo son exclusivamente poéticas, incluso algunas teñidas de meditación filosófica. Las críticas, como advertíamos, no tienen su blanco intencionado en la estructura de la sociedad del Antiguo Régimen, sino que van dirigidas contra las adulteraciones del sistema que han de ser reformadas. En este sentido, la apología de la ordenación social presidida por la unión entre monarquía y religión se repite constantemente. En definitiva, encontramos en este apartado todo el rico repertorio, común a la anterior generación periodística que, basado en el papel de la virtud social, exalta los ideales del catolicismo ilustrado. Tanto en estos temas como en el contexto general del periódico hubo de tener indudable influencia el peso de los sacerdotes suscritos.

3.- Temas de las «Luces». Son los dedicados expresamente a tratar de filosofía, crítica, religiosidad, educación, política, literatura, economía, agricultura y ciencias; en total, 254 páginas, de las que 114 se ocupan específicamente de medicina o temas relacionados con la salud pública. Dos hechos interesantes a constatar previamente. En primer lugar, ciertos temas sólo se abordan en el tercer volumen (filosofía, crítica, educación), cuando el periódico ha pasado la etapa inicial que, en cambio, requería auténticas declaraciones de principios (el número de páginas concedido al problema de la religiosidad es grande en el primer volumen). En segundo lugar, aquellos temas se abordan muy escuetamente y sin conflictividad: en la mayoría de los artículos los redactores no van más allá de lo que sería una definición basada en los planteamientos ilustrados oficiales. Razón de más para que los obviemos y nos dediquemos a señalar algunos rasgos concernientes a la actitud de los editores ante la ciencia. Ciertamente, no cabe esperar originalidades en este aspecto, pero sí una excelente información y una coherencia de fondo que da respuesta a la necesaria adecuación entre el contenido del periódico y la intencionalidad divulgativa.

Los presupuestos básicos de tal actitud se perfilan en dos artículos que hemos incluido en el apartado de «crítica». Uno de ellos comienza así: «la experiencia y la razón son los medios ciertos para el mejor progreso de las ciencias», para después pasar revista a la validez del principio de autoridad en las distintas ramas científicas ${ }^{(31)}$. Éste va a quedar totalmente excluido en las ciencias físicas y matemáticas, y sólo es aceptado, con las limitaciones que establece la crítica, en la retórica, jurisprudencia e historia. Dentro de esta última se postula como fundamental el principio del argumento negativo. Otro artículo en el mismo sentido insistía en la cuestión: la lectura de los escritores antiguos, que se reputan como 
falibles sin que por ello resulten despreciados, sólo puede hacerse con crítica y prudencia.

Pero sin duda es una polémica sostenida entre un colaborador que firmaba como El ecléctico y el editor Bado la que pone sobre el tapete las cuestiones más importantes. El primero remitió un «Aviso filosófico contra la filosófica preocupación de algunos aperipatéticos» ${ }^{(32)}$ que fue respondido contundentemente por Bado en una larga serie de artículos ${ }^{(33)}$. La polémica la suscitó un breve artículo en el que don Luis había tocado una fibra tremendamente sensible: ensalzar los progresos obtenidos por la filosofía de los modernos (entendida como estudio de la Naturaleza) y atacar el escolasticismo aristotélico ${ }^{(34)}$. El «Aviso filosófico» del Ecléctico respondió con una argumentación en la que pretendía demostrar la nula novedad de los modernos, que en su opinión sólo habían plagiado sus obras y descubrimientos de los autores antiguos sin reconocerles los méritos que les correspondían. Sorprende desde luego la cantidad de autores citados. Entre los modernos no hay ausencias importantes: Voltaire, Rousseau, Diderot, La Mettrie, Descartes, Bacon, Locke, Melebranche, Leibnitz, Buffon, Newton, Kepler, Gassendi, Franklin, Servet, Wotton, Harvey, Falopio y otros muchos.

Bado comenzaba su réplica denostando nuevamente las Escuelas por su inutilidad, que él mismo había sufrido, y acusando precisamente de plagio a su opositor: «pues el trabajo que $\mathrm{Vm}$. se ha tomado en copiar la obra que Mr. Dutens, dió a luz con el título de Reflexiones sobre el origen de los descubrimientos atribuidos a los modernos ha sido (...) inútil». Y en las cuestiones que se propone responder aparece neta la actitud ante la ciencia y el conocimiento propia de un ilustrado. Así, Bado no niega la importancia de la aportación realizada por la herencia clásica en la formación de la nueva filosofía; tampoco desprecia a Aristóteles, cuya genialidad reconoce, sino a las futilidades escolásticas. Sobre todo contra ellas dirige su respuesta, a la que da cariz autobiográfico. Es plenamente consciente de la línea que le separa del Ecléctico: la acusación fundamental que le dirige es la de fanatismo y cortedad de espíritu; al propio tiempo, sin hacer acopio de autores, Bado traza su propia tradición cultural escribiendo unos pocos nombres: Vives, Erasmo, Gasendi, Cano, Feijoó. el centro de la polémica no lo ocupan, pues, las consecuencias de impiedad que conllevaba la filosofía moderna, hacia las que El Ecléctico pretendía desplazarla (Bado también rechaza el ateísmo y el deísmo como consecuencias de errores de la razón humana), sino que gravitaba en torno al combate contra la escolástica, cuya desaparición tenía implicaciones no sólo metodológicas, sino también sociales.

En la misma línea podemos situar otros artículos, los de religiosidad, consagrados a la crítica de los errores populares y a las distintas formas de superstición, y una traducción resumida, «La existencia de Dios, y su Providencia, deducidas de las maravillas de la Naturaleza», de la obra de Neuwentyt publicada en 1725 y vulgarizada en Francia por el abate Pluche ${ }^{(35)}$. Se trata de sostener el viejo argumento ilustrado, defendido con firmeza por los redactores al diferenciar la «buena» y la «mala» filosofía, por el cual se afirma que el estudio de las ciencias nturales permite un mejor acercamiento a la sabiduría, la bondad y la grandeza del Creador. Una prueba, además, de las dificultades por las que pensaban los ilustrados murcianos para poder consolidar posiciones y conquistar terreno pro- 
pio frente a quienes defendían una concepción totalmente teológica. Los artículos referentes a física y medicina se tratarán al final.

4.-Colaboraciones. Mención aparte merecen los artículos remitidos al periódico por lectores y las polémicas que suscitaron, si bien la adscripción temática cabe realizarla sobre los anteriores apartados, dada su heterogeneidad y ausencia de una única línea. Incluso cabe la posibilidad que en tales disputas dialécticas se halle encartado algún redactor camuflado bajo seudónimo ${ }^{\left({ }^{(36}\right)}$. Por tanto, vuelven a plantearse aquí las mismas cuestiones anteriores: lucha contra la ignorancia religiosa, preocupación por la educación de los nobles ${ }^{(37)}$, crítica a los petimetres, así como unas curiosas polémicas entre El Curioso Preguntón y El Respondón Eterno, entre otros, de indudable interés, pero cuya reseña omitimos. Subrayemos únicamente que se basan en la obra del Dr. Miravete, El Espúdeo, de la que se ha ocupado en su comunicación M. Martínez Gomis.

5.-Asuntos varios. Completan la clasificación otros asuntos variados, entre los que destacan los dedicados a exaltar el espíritu patriótico ante la guerra con Francia. No obstante, las guerras y los duelos se juzgan negativamente como manifestaciones de la violencia humana.

Una sección de noticias de distinto tipo (ventas, compras, pérdidas, notas bibliográficas, reseñas de precios, suscripciones, etc.), precedidas habitualmente de unas breves líneas sobre vidas, anécdotas y apotegmas, cerraba cada número del Correo Literario.

Quizá sea en los artículos referentes a ciencias físicas y a la medicina y salud pública, que hemos dejado para el final, donde cabría buscar la mayor cantidad de trabajos originales, aun cuando éstos no se prodiguen excesivamente. No obstante, como indica Guinard, la inclusión de temas científicos en una proporción importante es ya de por sí una novedad ${ }^{(38)}$, dado lo poco frecuente de tales temas en la prensa española del siglo XVIII. La presencia entre los redactores del Correo... de un matemático-físico y de un médico hubo de animar la escena. Con todo, como decíamos, los temas médicos y de salud pública ocupan la parte más importante. Prácticamente, nos limitaremos a enumerar los asuntos científicos tratados:

- Un discurso sobre el régimen de conservar la salud ${ }^{(39)}$. Estudio impregnado de fuertes influencias sensualistas, estructurado sobre bases comparativas, que contempla la influencia de la conducta y los hábitos sociales en la génesis de las enfermedades del individuo. Contiene elementos típicamente ilustrados: rechazo del método deductivo aristotélico, exlusión de las argumentaciones fundadas en principios morales, bondad de la naturaleza y de la conducta de los salvajes, etc. Este discurso fue replicado, en tono casticista, pero sin razones de consideración, por El Tío Antón Terrones.

- Nota sobre una memoria de Jaime Menós de Llena (a la sazón suscriptor) sobre la curación de la perlesía resultante de las apoplejías. En cuanto a la salvación del afectado, siguiendo a Hipócrates, recomienda la sangría. Para la curación de la parálisis, «la reiterada urticación del pesqueso (sic) a la rabadilla, y a toda la parte paralítica por el espacio de un mes» efectuada con «vegetomineral fresco, frontando las partes, $y$ dexando sobre ellas unos paños mojados; pur- 
gando por Epicrisim todos los días, acompañando con el purgante los polvos de la corteza del Quarango, el nitro depurado y con el oximel simple hago un electuario». Dice haber sanado en Barcelona a diez sujetos ${ }^{(40)}$.

- Discurso sobre la obligación que tienen las madres de criar a sus hijos ${ }^{(41)}$. Obviamente, en la línea de preocupación demográfica de los ilustrados y, por supuesto, no dirigido a las matronas de humilde condición.

- Discurso contra la costumbre de enterrar a los cadáveres en los templos ${ }^{(42)}$. Contiene razones médicas en contra de esta práctica, centradas sobre todo en la corrupción que se origina en el aire; combinado con notas históricas.

- Discurso político sobre la despoblación de España, ampliado después a Europa. Las causas de la misma se atribuyen fundamentalmente a la ignorancia: obstáculos a la generación, crecimiento y maduración del hombre hasta el momento del nacimiento (vida claustral); y obstáculos que abrevian la vida tras el nacimiento. Este discurso se concibe de modo semejante al del régimen de conservar la salud, sobre las enfermedades originadas por la conducta social, ahora enfocado en las prácticas que son fruto de la ignorancia ${ }^{(43)}$.

- Memoria del Dr. Jaime Menós contra la inoculación de las viruelas (notaresumen de la publicada por Menós en 1785). Los conocidos argumentos de este médico también fueron contestados en el Correo por Juan de Alegre, quien dice estar practicando la inoculación desde hacía tiempo con total éxito ${ }^{(44)}$.

- Unas Reflexiones sobre los efectos del aire relativas a los negros, sin firmar (atribuible, pues, a los editores). Entre otras, recoge la tesis de que los rasgos propios de la raza negra son producto de una adaptación al medio ${ }^{(45)}$.

Por lo referente a la física es mucho menor el espacio que se le concede: una discusión física ${ }^{(46)}$ sobre la observación de una tormenta y la comprobación empírica de los efectos producidos sobre la torre de la catedral de Murcia por la caída de uno de los rayos. Las referencias a Franklin son abundantes. También, una nota sobre una obra de Cayetano María Menós de Romeo sobre el temor a los rayos, en realidad no catalogable como obra científica: el autor, en su afán por combatir las máximas de los philosophes, viene a asegurar que últimamente caían en Barcelona menos rayos por las «sabias disposiciones» espirituales tomadas por el obispo. Esta nota constituye la única salida de tono del Correo en estos números respecto de sus planteamientos científicos ${ }^{\left({ }^{47}\right)}$. Por último, registremos un artículo sobre la observación de una posible aurora boreal en la región murciana ${ }^{(48)}$ y unos sueltos sobre el carácter y definición de la física, de escasa extensión.

Concluye aquí la aproximación que nos proponíamos efectuar a la prensa murciana del siglo XVIII y en concreto al Correo Literario. No es preciso resaltar que, por la propia forma en la que concebimos el trabajo, cuanto se ha afirmado viene marcado por el sello de cierta provisionalidad, y que puede y debe ser revisado y examinado a la luz de investigaciones más detalladas. Razón por la cual también nos privamos de formular una enumeración de conclusiones.

Ahora bien, sí deseamos efectuar, para finalizar, algunas consideraciones. En primer lugar, y ante todo, insistir en el esquema pergeñado al principio, valorando el hasta hoy casi ignorado peso que tuvo el Seminario de San Fulgencio, como foco ilustrado-jansenista y como institución universitaria de primerísimo 
orden, no sólo murciano, sino nacional, en el contexto de nuestras Luces. También para un estudio más profundo de la prensa y de la Ilustración en Murcia, el Seminario, su obra y sus profesores deberá convertirse en uno de los primeros polos de referencia a tener muy presente.

En segundo lugar, coincidimos de forma prácticamente plena con J. Hernández Franco respecto de la contemplación de la prensa ilustrada murciana, él desde el estudio del Diario, y nosotros desde el Correo. En este sentido, el Correo también es un periódico conservador. Ahora bien, precisando el sentido del término si no queremos caer en la indefinición o incluso el anacronismo. Conservador, como bien señalaba Hernández Franco a propósito del Diario, porque la cultura ilustrada, por naturaleza, era esencialmente elitista y, en rasgos generales, no se planteaba una reforma estructural del sistema social y político del Antiguo Régimen. Conservador, también, porque como obra periódica, así se lo exigiría la mayoría de su público, entre el cual el umbral que daba entrada a las auténticas Luces en muchos casos quizá no había sido traspasado, y en otros muchos éstas no eran mucho más que un pálido eco que llegaba amortiguado con los visos de simple «aire de los tiempos». Pero estamos convencidos de que los redactores, y sobre todo Luis Santiago Bado, podían haber llegado ciertamente más lejos en otro medio de expresión o en otro contexto social e histórico. No nos vale en esto que Bado fuese después un declarado absolutista. La verdadera lucha no estaba planteada contra la impía filosofía, sino contra la tenaz vitalidad de la superstición y de la escolástica al uso. Y en este sentido, con todas las concesiones que se quieran, los editores del Correo hicieron gala de una solidez de principios ciertamente encomiable.

$Y$ en tercer lugar, relacionado con lo anterior, también manifestaron una versatilidad de adaptación del mensaje al medio y al contexto siempre digna de apreciarse en todo periodista. Aunque hecho de pasos cortos, es fácilmente observable un cierto proceso de maduración en el conjunto de los números del Correo, máxime si los comparamos con los del Diario. 


\section{CUADRO I \\ IMPLANTACIÓN GEOGRÁFICA Y SOCIOPROFESIONAL}

\begin{tabular}{|c|c|c|c|c|c|c|c|}
\hline GRUPOS $^{\text {ZONAS }}$ & $\begin{array}{l}\text { MURCIA } \\
\text { Y REINO } \\
\end{array}$ & ANDALUCIA & $\begin{array}{l}\text { MADRID } \\
\text { CASTILLA }\end{array}$ & $\begin{array}{l}\text { VALENCIA } \\
\text { Y REINO }\end{array}$ & CATALUÑ & R. E. $\left({ }^{*}\right)$ & TOTALES \\
\hline $\begin{array}{l}\text { Oficios y cargos } \\
\text { municipales } . . \ldots . . .\end{array}$ & 6 & 1 & - & - & - & 1 & 7 \\
\hline $\begin{array}{l}\text { Funcionarios } \\
\text { administración }\end{array}$ & 12 & 9 & 4 & 4 & - & 17 & 29 \\
\hline Correos................... & 1 & - & - & - & - & - & 1 \\
\hline $\begin{array}{l}\text { Nobles con } \\
\text { título }\end{array}$ & 2 & 5 & 4 & 7 & 2 & 18 & 20 \\
\hline Militares . & 5 & 3 & 1 & - & 4 & 8 & 13 \\
\hline Cónsules ............ & 1 & - & - & 1 & - & 1 & 2 \\
\hline Comerciantes .... & 12 & 3 & 3 & 1 & 1 & 8 & 20 \\
\hline Clero secular & & & & & & & \\
\hline y catedralicio .... & 36 & 9 & - & 6 & 2 & $\begin{array}{r}17 \\
8\end{array}$ & $\begin{array}{l}53 \\
17\end{array}$ \\
\hline Clero regular..... & 9 & $\begin{array}{l}3 \\
2\end{array}$ & 1 & 1 & 3 & $\begin{array}{l}8 \\
5\end{array}$ & 7 \\
\hline $\begin{array}{l}\text { Docentes........... } \\
\text { Colegiales }\end{array}$ & $\begin{array}{l}2 \\
1\end{array}$ & $\begin{array}{l}2 \\
1\end{array}$ & -3 & $\overline{1}$ & - & 2 & 3 \\
\hline $\begin{array}{l}\text { Colegiales ..... } \\
\text { Funcionarios }\end{array}$ & 1 & 1 & & 1 & & & \\
\hline $\begin{array}{l}\text { Funcionarios } \\
\text { inquisición..... }\end{array}$ & 2 & - & 1 & - & - & 1 & 3 \\
\hline Médicos .............. & 2 & - & - & - & 1 & 1 & 3 \\
\hline Notarios & - & - & - & - & 1 & 1 & 1 \\
\hline Bibliotecas.......... & 1 & - & - & - & - & - & 1 \\
\hline Impresores.......... & 2 & - & - & - & - & - & 2 \\
\hline Cafés ...................... & 1 & - & - & 一 & - & - & 1 \\
\hline Sin especificar... & 56 & 25 & 13 & 8 & 19 & 65 & 121 \\
\hline Totales/Grupos. & 151 & 61 & 30 & 29 & 33 & 153 & 304 \\
\hline
\end{tabular}

R. E. $\left({ }^{*}\right)$. RESTO DE ESPAÑA (Totales de suscriptores sin incluir Murcia y su reino). 


\section{CUADRO II \\ CLASIFICACIÓN DE ARTÍCULOS}

\begin{tabular}{|c|c|c|c|c|c|}
\hline GRUPOS & томо I & томо II & TOMO III & $\begin{array}{r}\text { TOT } \\
\text { PARCIAL }\end{array}$ & TES \\
\hline Historia & & & & & 207 \\
\hline - de Murcia & 54 & 67 & 53 & 174 & \\
\hline - crítica histórica y otros & 6 & 20 & 7 & 33 & \\
\hline Composiciones en verso & & & & & 184 \\
\hline - sátiras, letrillas, sonetos & 27 & 21 & 2 & 50 & \\
\hline - fábulas & 40 & 31 & 27 & 98 & \\
\hline - temas filosóficos y poéticos & 19 & 6 & 11 & 36 & \\
\hline Temas de las Luces & & & & & 254 \\
\hline - filosofía & 0 & 0 & 50 & 50 & \\
\hline - crítica & 0 & 0 & 7 & 7 & \\
\hline - religiosidad y afínes & 23 & 3 & 0 & 26 & \\
\hline - educación & 0 & 0 & 4 & 4 & \\
\hline - política & 5 & 10 & 3 & 18 & \\
\hline - literatura & 0 & 0 & 3 & 3 & \\
\hline - economía & 6 & 0 & 0 & 6 & \\
\hline - agricultura & 0 & 6 & 0 & 6 & \\
\hline - física & 9 & 3 & 8 & 20 & \\
\hline - medicina y salud pública & 24 & 56 & 34 & 114 & \\
\hline Colaboraciones & 82 & 57 & 49 & 188 & 188 \\
\hline Otros temas & 0 & 1 & 39 & 40 & 40 \\
\hline Anéc., apoteg., vidas, hechos $(*)$ & 10 & 0 & 12 & $10 *$ & $10^{*}$ \\
\hline Noticias $(*)$ & - & - & - & $15 *$ & $15^{*}$ \\
\hline $\begin{array}{l}\text { Sostenimiento económico, sus- } \\
\text { cripciones, etc. }\left({ }^{*}\right)\end{array}$ & - & - & - & $20 *$ & $20 *$ \\
\hline & & & & 918 & 918 \\
\hline
\end{tabular}

TOTAL CONTABILIZADO: 918 páginas.

TOTAL REAL DE PÁGINAS: 855

Error: 63 páginas, $7,37 \%$ aprox.

(*) Los apartados con asterisco proporcionan una estimación aproximada del número de páginas: se han contabilizado, cuando se ha hecho, el número de artículos. En todos los casos, se trata de breves párrafos (5-6 líneas) que jamás alcanzan más de la media página. 


\section{NOTAS}

(1) Este trabajo se presentó como comunicación al VII Congreso Nacional de Historia de la Medicina, celebrado en Alicante los días 6 a 9 de abril de 1983, y hasta ahora ha permanecido inédito. Sólo hemos revisado, actualizándolas, algunas pequeñas partes del texto original y de las notas bibliográficas.

(2) Los tomos del Correo se encuentran en el Archivo Municipal de Murcia. Falta en él el tomo VIII, que localizamos después de presentar la comunicación entre los fondos de la Biblioteca Nacional de Madrid, donde la colección de este periódico se encuentra completa.

(3) Hecemos gracia al lector de efectuar un inventario de las señeras aportaciones historiográficas que se han realizado. Nos limitaremos a citar los trabajos del profesor F. LÓPEZ, «Aspectos específicos de la Ilustración española», en II Simposio sobre el P. Feijoo y su Siglo, Oviedo, 1981, I, pp. 23-29; y «Rasgos peculiares de la Ilustración en España», en Mayans y la Ilustración. Simposio Internacional en el Bicentenario de la muerte de Gregorio Mayans, Valencia-Oliva, 1981, II, p. 629671 , por considerarlos muy a propósito para una introducción al problema que aquí nos ocupa.

(4) JEAN SARRAILH, La España ilustrada de la segunda mitad del siglo XVIII, Madrid 1957, pp. 122-123.

(5) PAUL-JACQUES GUINARD, La presse espagnole de 1737 a 1791. Formation et signification d'un genre. C. H. R., 1973, p. 13.

(6) Ibid., p. 14-15.

(7) Ibid., p. 14.

(8) Vid. RICHARD HERR: España y la revolución del siglo XVIII, Madrid 1964.

(9) M. a DOLORES SAIZ: Historia del periodismo en España. 1. Los orígenes. El siglo XVIII. Madrid 1983. Únicamente alude al Diario de Murcia, inmediato predecesor del Correo, pero omite por entero este último, pese a tener mucha mayor entidad, desde todos los conceptos, que el Diario.

(10) Fundamentalmente, PÍO TEJERA: Biblioteca del murciano, 3 tomos, Madrid 1941-1957; JOSE BALLESTER NICOLÁS: Amanecer de la prensa diaria en Murcia, Murcia 1971; y JUAN HERNÁNDEZ FRANCO, «El Diario de Murcia: estudio analítico-social de un periódico ilustrado», Anales Universidad Murcia. Filosofía y Letras, XXXVIII, n. ${ }^{\circ} 4,1979-1980$, pp. 46-49.

(11) R. HERR: Op. cit., cap. VI.

(12) El 18 de diciembre de 1986 tuvo lugar la lectura de nuestra tesis doctoral, que versaba precisamente sobre Jansenismo y reforma educativa en la España ilustrada: el Seminario de San Fulgencio de Murcia (inédita). Publicaciones sobre el Seminario, entre otras, pueden verse: FERNANDO JIMÉNEZ DE GREGORIO, «El Colegio-Seminario conciliar de San Fulgencio de Murcia», Anales Universidad Murcia, vol. III, 1949-1950; CAYETANO MAS GALVAÑ , Anales de la Universidad de Alicante. Historia Moderna, n. ${ }^{\circ} 2,1982$, pp. 259-290; y ANTONIO VIÑAO FRAGO, «El ColegioSeminario de San Fulgencio: Ilustración, liberalismo e Inquisición», Areas, 6, Murcia, 1986, pp. 17-48

(13) Vid. FRA NCISCO J. FLORES ARROYUELO, Sociedad Murciana e Ilustración. Murcia, 1977; y MATÍAS VELÁZQUEZ, Génesis y primeros años de la Real Sociedad Económica de Amigos del País de Murcia, Tesina de licenciatura inédita, Universidad de Murcia, 1984 (consultada gracias a la gentileza del autor).

(14) Conocemos referencias a la existencia de otras publicaciones periódicas en Murcia, muy efimeras. Así J. P. GUINARD, Op. cit., p. 201 habla de un Semanero de Murcia, que nosotros no hemos visto y del que él confiesa tener sólo referencias indirectas. 
(15) P. TEJERA: Op. cit., T. II, pp. 292 y ss. Son los mismos redactores del Diario: vid. J. HERNÁNDEZ FRANCO, Op. cit., p. 51.

(16) P. TEJERA: $O p$. cit., T. II, pp. 434 y ss.

(17) Ibid., T. II, pp. 231 y ss.

(18) Ibid., T. II, pp. 434 y ss.

(19) P. GUINARD: Op. cit., p. 37.

(20) TEJERA, T. II, p. 634 y también J. HERNÁNDEZ FRANCO: Op. cit., pp. 51-52.

(21) TEJERA: loc. cit.

(22) HERR: Op. cit., p. 292.

(23) A mero título de referencia, véase $M .{ }^{\text {a }}$ TERESA PÉREZ PICAZO y GUY LEMEUNIER, El proceso de modernización de la región murciana (siglos XVI-XIX), Murcia 1984, y el volumen VII de la Historia de la región murciana, Murcia 1984.

(24) GUINARD, p. 64 y ss.

(25) A este efecto, podían contar con los suscriptores del Diario, cuya lista publica HERNÁNDEZ FRANCO en el referido trabajo.

(26) Según las noticias publicadas en el propio periódico en números sucesivos.

(27) Listas de suscriptores publicadas al final de cada número del periódico.

(28) GUINARD, pp. 367 y ss.

(29) Si el siglo XVIII fue el siglo de la Historia en España, también cobra renovado auge el cultivo de esta disciplina en Murcia. Vid., FRANCISCO JAVIER DÍEZ DE REVENGA y M. ${ }^{a}$ DoLORES EGEA MARCOS, «La imprenta y los impresores en la Murcia del siglo XVIII», en Francisco Salzillo y el Reino de Murcia en el siglo XVIII, Murcia 1983; y M. ${ }^{a}$ DOLORES EGEA y M. ${ }^{a}$ CONCEPCIÓN RUIZ ABELLÁN, El libro en Murcia en el siglo XVIII, Madrid 1985.

(30) GUINARD: parte III, cap. II.

(31) Número 77, tomo III, pp. 53 y ss.

(32) Núm. 91, t. III, pp. 162 y ss.

(33) Núms. 99, 103 y 105.

(34) Núm. 84, pp. 107 y ss.

(35) J. SARRAILH, pp. 503-504.

(36) Muy posiblemente sea así, dado lo extendido de la costumbre en la época, de fingir los redactores cartas de lectores, siguiendo el modelo del Spectator y sus imitadores. Pero es difícil, en el nivel en que hemos planteado nuestro trabajo, delimitar en qué casos ocurre esto, dado que en otros muchos casos la autenticidad de los remitidos parece incuestionable.

(37) En Murcia se planteó, y desechó, un proyecto de creación de un colegio de nobles. Cf. JIMENEZ DE GREGORIO, loc, cit.

(38) GUINARD, p. 481.

(39) Núm. 5, t. I, pp. 35 y ss.

(40) Núm. 22, t. I, pp. 170 y ss.

(41) Núm. 36, t. II, pp. 4 y ss.

(42) Núm. 38, t. II, pp. 22 y ss.

(43) Núm. 55, t. II, pp. 156 y ss.

(44) Núm. 60, t. II, pp. 195 y ss.; Núm. 71, t. III, pp. 5 y ss.

(45) Núm. 89, t. III, pp. 149 y ss.

(46) Núm. 7, t. I, pp. 53 y ss.

(47) Núm. 31 , t. 1, pp. 247 y ss.

(48) Núm. 37, t. II, pp. 10 y ss. 